\title{
Flexible patches for mm-wave holography
}

\author{
J. Burch, ${ }^{1}$ J. Ma, ${ }^{1,2}$ R.I. Hunter, ${ }^{1}$ S.A. Schulz, ${ }^{1}$ D.A. Robertson, ${ }^{1}$ G.M. Smith, ${ }^{1}$ J. Wang, ${ }^{2}$ and A. Di Falco ${ }^{1, a)}$ \\ ${ }^{1)}$ School of Physics and Astronomy, University of St Andrews, North Haugh, St Andrews, KY16 9SS, \\ $U K$ \\ ${ }^{2)}$ School of Science, Jiangnan University, 1800 Lihu Avenue, Wuxi, Jiangsu, 214122, China
}

(Dated: 25 June 2019)

In this work, we demonstrate, in simulation and experiment, reflection metasurface holograms operating in the millimeter wavelength range. Flexible holographic metasurface patches are the ideal platform to retrofit existing millimeter wavelength equipment to enhance and extend their functionality, e.g. for antennas and electromagnetic shielding. We present both a rigid and flexible implementation of the holographic metasurfaces, where the meta-atom is based on a three-layered structure with a gold c-ring as the polarization conversion element.

Holographic metasurfaces (HMSs) are the ideal platform for controlling light, enabling efficient scattering engineering ${ }^{1-6}$, multiplexing ${ }^{7-15}$, three-dimensional holography ${ }^{16}$. The underpinning principle of HMSs is the use of meta-atoms to determine the properties of the scattered light. The meta-atom is the building block of the HMS and through its shape and/or orientation allows controlling point-by-point both the phase and the amplitude of scattered light. Typically, HMSs have been realized in the visible wavelength range, with other notable results in the near infrared $^{17}$ and ultraviolet ranges ${ }^{18,19}$. At longer wavelengths, in the gigahertz and terahertz wavelength ranges, there have also been significant proposals and experimental results for applications of metasurfaces in lensing ${ }^{20-22}$, sensing ${ }^{23,24}$, tunable response $\mathrm{e}^{25-29}$, cloaking $^{30-32}$, controlling phase and amplitude $^{33,34}$, absorbers ${ }^{35}$, and polarization conversion devices $^{36}$. Progress has also already been made on rigid HMS designs with the creation of a frequency multiplexed transmission $\mathrm{HMS}^{37}$, a broadband transmission vortex generation $\mathrm{HMS}^{38}$.

To create a HMS we first need to recover the phase distribution of the required holographic image. Typically, this step is performed using an iterative phase reconstruction algorithm, such as the Gerchberg-Saxton algorithm (GSA) ${ }^{39}$. The GSA requires propagating light backward and forward between the HMS and holographic image planes. The standard Fourier transform is the most commonly used propagator, which is well suited to deal with flat metasurfaces and imaging planes ${ }^{40}$, whereas for arbitrarily shaped holographic and imaging planes, it is necessary to use a more sophisticated propagator, e.g. based on the Rayleigh-Sommerfeld equation $^{41-43}$.

The millimeter wavelength range is vital for applications in broadband telecommunications (e.g. 5G), radar (e.g. weapons systems, terrain monitoring, cloud radar and security screening), radiometry (e.g. earth resource studies, cosmic background), and magnetic resonance (e.g. fusion heating and diagnostics, electron spin resonance and dynamic nuclear polarization). It offers a compromise between the microwave and the visible/infrared wavelength ranges exhibiting comparatively low atmospheric attenuation, relative to microwave

\footnotetext{
a)Electronic mail:

adf10@st-andrews.ac.uk; https://synthopt.wp.st-
}

systems, but maintaining a higher imaging resolution and offering higher bandwidths. As a result, millimeter wavelength range equipment is commonplace. Traditionally, when the functionality of this equipment requires enhancing or extending, e.g. to modify a $4 \mathrm{G}$ base station antenna, it must be replaced which is expensive and wasteful. As a result, the ability to modify or enhance the electromagnetic properties of a device by retrofitting it with a patch is an extremely attractive idea.

HMS patches are the ideal way to retrofit these millimeter wavelength systems because they are essentially twodimensional due to having a sub-wavelength thickness. This makes HMSs comparatively easy to fabricate and limits their losses compared to three-dimensional metamaterials. Furthermore, two-dimensional HMSs are ideally exploited in flexible implementations which offer a further opportunity for post-fabrication tunability ${ }^{9,10}$ and enable large scale fabrication routes ${ }^{44}$. Most importantly, flexible HMSs can be applied to non-flat substrates ${ }^{42,43,45}$, and can be used to transfer an engineered photonic skin to any device that can not be directly micro-fabricated and/or made of material without a suitable photonic response.

Here, we demonstrate the experimental realization of rigid and flexible HMS, which could be retrofitted to existing millimeter wavelength equipment, operating at a wavelength of $\lambda=3.19 \mathrm{~mm}(94 \mathrm{GHz})$.

For the HMS we chose a three-layer design, where the meta-atoms are separated from a back-reflection plane by a dielectric spacer. This is a well accepted solution that guarantees a high phase conversion efficiency between the incident and reflected beam. 1,7,11,42,43

We designed the meta-atoms with the CST Microwave Studio simulation package, by sweeping through the relevant parameters for a standard c-ring design ${ }^{20}$. In figure 1 we show a typical meta-atom, with the relevant geometrical parameters. From these simulations, we created a parameter look-up table, which maps a specific shape to the desired de-phasing.

The bounds of the parameter space are determined by different factors. The meta-atoms pitch, $p$, needs to be kept subwavelength to avoid exciting high diffraction orders, which would limit the efficiency ${ }^{7}$. A very small pitch would instead strongly couple the meta-atoms, which in turn would require modeling the entire HMS, which is computationally extremely expensive. To reduce the computational and design effort, we quantized the phase in 30 degree increments. 
(a)

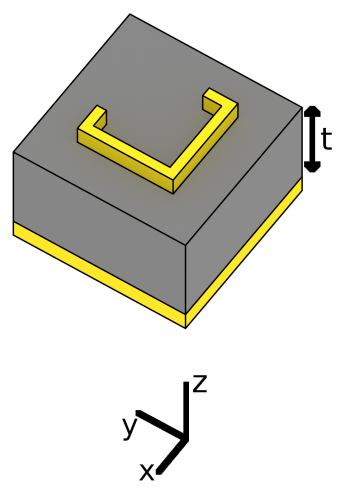

(b)

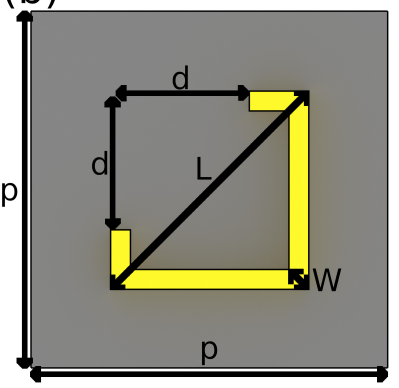

(c)

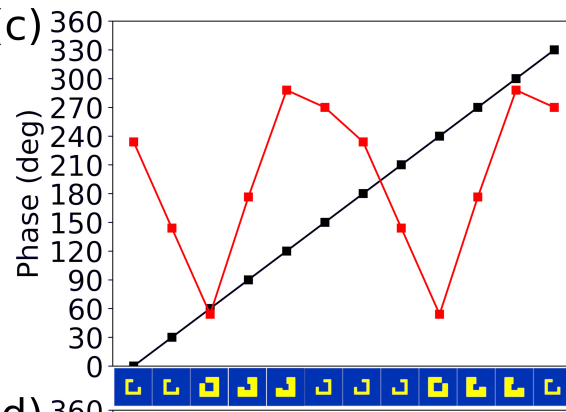

(d) 360

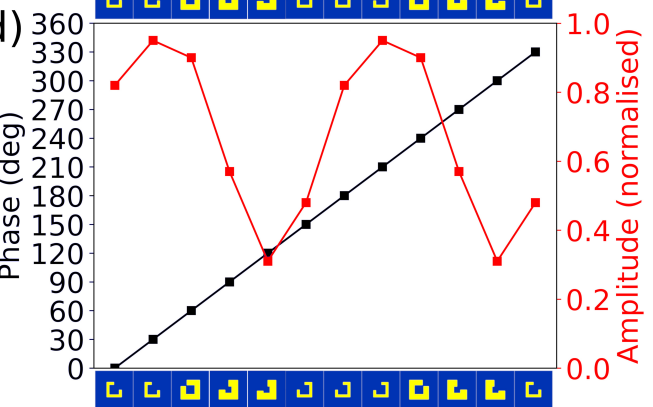

FIG. 1. (a) A diagram of the unit cell structure of a c-ring. The spacing between the c-ring and the backplane is denoted as $t$. (b) The same unit cell as seen from the top. The pitch is defined as $p$, the c-ring is centered, the parameters $\mathrm{L}, \mathrm{d}$, and $\mathrm{W}$ are defined as in the diagram. This meta-atom is left-handed, which means that the cutout is on the left side. Simulated de-phasing and normalized amplitude for each meta-atom at $\lambda=3.19 \mathrm{~mm}$, where the dielectric spacer is (c) rigid silicon with a pitch of $650 \mu$ and (d) flexible PMMA with a pitch of $800 \mu \mathrm{m}$. The de-phasing between the illustrated meta-atoms is 30 degrees. The drawn meta-atoms are for illustration and do not necessarily represent the actual meta-atoms.

As such, we required only 12 unique meta-atoms to cover the $0 \rightarrow 360$ degree range. This quantization level is standard in HMSs $1,7,11,42,43$. However, we can reduce this requirement further to a 6 meta-atoms library through a symmetry argument, as the corresponding meta-atom parameters for the phase range of $180 \rightarrow 360$ degrees are identical to those for $0 \rightarrow 180$ degrees but flipped through the $x$ axis. This is shown in figure 1.

For the CST simulations, we used unit cell boundary conditions for the $x$ and $y$ axes and open boundary conditions for the $z$ axis. The input electric field was linearly polarized along the $x$ axis and traveling in the $-z$ direction. The output electric field was linearly polarized along the $y$ axis and traveling in the $z$ direction. The electrical conductivity of the gold was taken as $45.61 \mathrm{MS} / \mathrm{m}$, and the permeability was taken as $\mu=1$, from the standard CST material properties library. With this in mind, we designed suitable meta-atoms for two implementations, with silicon and PMMA as spacing layers, for the rigid and flexible case, respectively. In both cases the gold thickness of both the c-rings and of the reflecting back-planes were set at $200 \mathrm{~nm}$. Ideally the thickness of the dielectric spacer should be chosen with an optical thickness approaching $\lambda / 4$. However, due to experimental constraints, we designed the meta-atoms for a low-loss silicon sample of thickness $t=115 \mu \mathrm{m}$ and a commercially available sheet of PMMA with thickness $t=400 \mu \mathrm{m}$. For the silicon we used $\varepsilon=11.670+0.004 j$ (from CST material properties library). The permittivity for the PMMA was taken as $\varepsilon=2.200$ (as extrapolated from the PMMA datasheet from Microchem). For these two implementations we obtain the meta-atom parame- ters displayed in tables I and II.

TABLE I. Meta-atom parameters for gold c-rings with a pitch of $650 \mu \mathbf{m}$ on silicon

\begin{tabular}{c|cccc} 
De-phasing $(\mathrm{deg})$ & $\mathrm{L}(\mu \mathrm{m})$ & $\mathrm{d}(\mu \mathrm{m})$ & $\mathrm{W}(\mu \mathrm{m})$ & Cutout side \\
\hline 0 & 350.0 & 128.3 & 60.0 & right \\
30 & 350.0 & 134.6 & 60.0 & right \\
60 & 450.0 & 102.0 & 120.0 & left \\
90 & 450.0 & 159.9 & 150.0 & left \\
120 & 450.0 & 177.2 & 150.0 & left \\
150 & 350.0 & 124.9 & 60.0 & left
\end{tabular}

TABLE II. Meta-atom parameters for gold c-rings with a pitch of $800 \mu \mathrm{m}$ on PMMA

\begin{tabular}{c|cccc} 
De-phasing $(\mathrm{deg})$ & $\mathrm{L}(\mu \mathrm{m})$ & $\mathrm{d}(\mu \mathrm{m})$ & $\mathrm{W}(\mu \mathrm{m})$ & Cutout side \\
\hline 0 & 550.0 & 60.0 & 60.0 & left \\
30 & 550.0 & 60.5 & 60.0 & left \\
60 & 550.0 & 79.0 & 80.0 & left \\
90 & 550.0 & 83.5 & 100.0 & left \\
120 & 550.0 & 94.2 & 100.0 & left \\
150 & 550.0 & 206.2 & 40.0 & right
\end{tabular}

The amplitude and de-phasing values for each of these meta-atoms are displayed in figure 1(c-d). The amplitude values of the meta-atoms are mostly above 50\%. The low amplitude values of some meta-atoms derives mostly from the requirement to avoid a strong coupling between meta-atoms and from the not ideal thickness of the dielectric layers. Both these limitations can be removed by simulating in full the coupling 
of differently shaped meta-atoms and using different spacers.

The fabrication process for the rigid and flexible implementation are displayed in figure $2(\mathrm{a}-\mathrm{c})$ and figure $2(\mathrm{~d}-\mathrm{g})$. For the rigid implementation, we deposited via e-beam evaporation $200 \mathrm{~nm}$ of gold onto both sides of a loss silicon dielectric spacer. Next, we spin-coated the sample with a $500 \mathrm{~nm}$ thick layer of SU-8 2000.5 (Microchem), followed by a preexposure baking at $90^{\circ} \mathrm{C}$ for 4 min and a standard electron beam lithographic step with a RAITH eLine Plus at $30 \mathrm{keV}$. After post-exposure baking at $90^{\circ} \mathrm{C}$ for $2 \mathrm{~min}$, the resist was developed with Ethyl Lactate (EC, Microchem) for $45 \mathrm{~s}$ and the pattern transferred onto the gold layer with an Ar based reactive ion dry etching step.

The fabrication of the flexible samples was completed in two steps. First, we evaporated $200 \mathrm{~nm}$ of gold onto one side of the $t=400 \mu \mathrm{m}$ thick PMMA dielectric spacer. We then spin-coated on a rigid carrier $\mathrm{a} \approx 20 \mathrm{~nm}$ thick sacrificial layer of Omnicoat (Microchem), followed by a $6.5 \mu \mathrm{m}$ thick layer of SU-8, which was crosslinked with a long UV flood exposure and standard post-exposure bake. The definition of the gold meta-atoms followed a similar procedure used for the rigid case. Finally, we lifted-off the SU8 membrane by dissolving the Omnicoat in a tetramethylammonium hydroxide based solution (MF319, Microchem) and placed it on the PMMA substrate.

(a)

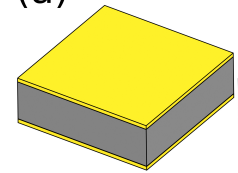

(b)

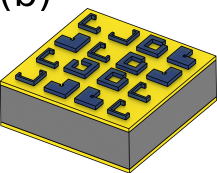

(e)

(d)

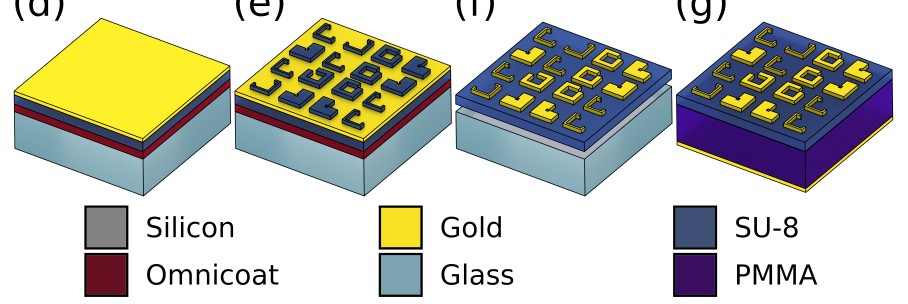

FIG. 2. Fabrication steps for the rigid (a-c) and flexible (d-g) implementations of our HMS. (a) Gold is evaporated onto both sides of the silicon (b) c-rings are lithography defined in resist (c) a dry etch defines the c-rings in the gold. (d) Glass is coated in a lift-off layer, then a polymer layer, then gold (e) c-rings are lithography defined in resist (f) a dry etch defines the c-rings in the gold, lift-off layer is dissolved ( $\mathrm{g}$ ) the lifted-off sample is combined with a PMMA substrate. The PMMA substrate has had gold evaporated onto the bottom side of it.

The fabricated samples for both the rigid and flexible implementations are displayed in figure 3 .

To demonstrate the operation of the HMSs, we designed the hologram to produce a focussed spot reflected at a predetermined angle, when excited at normal incidence. For the GSA we set the distance along $z$ between the hologram and the holographic image at $125 \mathrm{~mm}$, the hologram resolution was $30 \times 30$ pixels, the holographic image size was $100 \times 100 \mathrm{~mm}^{2}$, sampled with a $0.5 \mathrm{~mm}$ pitch. In the design,
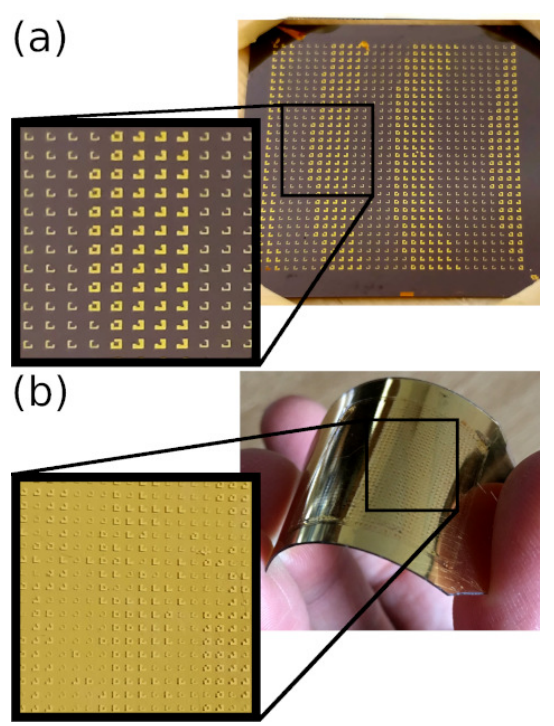

FIG. 3. Photographs, with expanded inserts, displaying the fabricated (a) rigid and (b) flexible implementations of our millimeter wavelength range flexible HMS. The pitch between meta-atoms is (a) $650 \mu \mathrm{m}$ and (a) $800 \mu \mathrm{m}$ respectively.

for the incident beam we used a Gaussian intensity distribution, with FWHM equal to $12.5 \mathrm{~mm}$, with a flat phase front, in accordance with the experimental source. The recovered phase distribution were eventually mapped onto a distribution of meta-atoms, with $p=650 \mu \mathrm{m}$ and $p=800 \mu \mathrm{m}$ for the rigid and flexible cases respectively, taking into account the amplitude of each unique quantized meta-atom.

To verify our fabricated samples in an experiment we used the setup described in figure 4 .

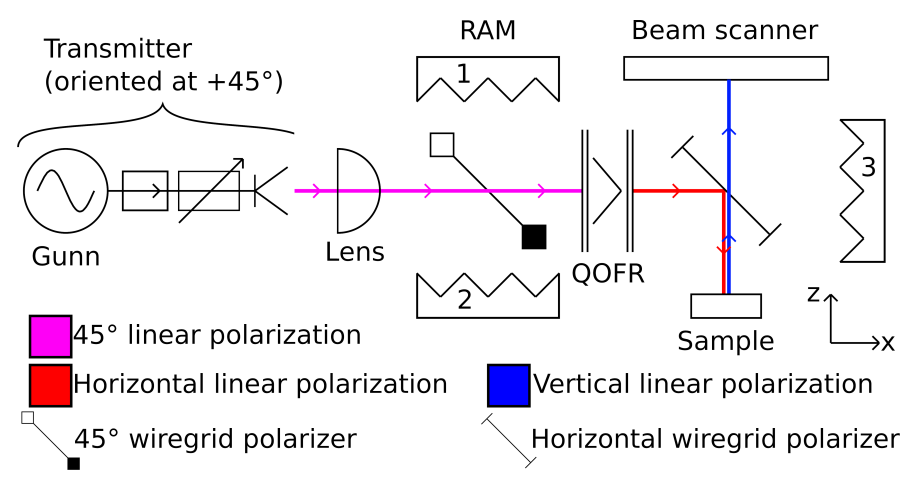

FIG. 4. A schematic of the experimental setup used to collect our holographic images.

Radiation at a wavelength of $\lambda=3.19 \mathrm{~mm}(94 \mathrm{GHz})$ was emitted by a millimeter wavelength Gunn oscillator into a waveguide isolator followed by a variable attenuator, which was coupled to free space via a corrugated feedhorn. The beam was collimated by a suitable lens, creating a beam with a Gaussian intensity profile and an approximately planar phase front at the plane of the sample. The beam then passed through a combination of a wire grid beam splitter and a quasi-optical Faraday Rotator (QOFR) to set the beam into 
the horizontal polarization and remove any unwanted radiation by dumping it into two blocks of radar absorbing material (RAM 1 and RAM 2). The beam was then sent on to a polarizing beam-splitter, which directed horizontally linearly polarized light onto the HMS at normal incidence, and dumped unconverted radiation into RAM3. The HMS created an holographic image with rotated polarization (vertical direction), which reached the detector unimpeded by the wire grid polarizer. The detector was a short section of single mode waveguide mounted on a motorized stage and coupled to a heterodyne receiver. The intermediate frequency (IF) signal from the receiver was measured with a spectrum analyzer. The beam scanner recorded the power as a function of the spatial position over an area of $100 \times 100 \mathrm{~mm}^{2}$ at a distance of $125 \mathrm{~mm}$ from the HMS

The results are displayed in figure 5. In figure 5(a), we show the image from a simple polarization rotation mirror to find the size and quality of the collimated beam. In figure 5(be), we show our deflected and focussed holographic image in simulation and experiment, which are in good agreement. The small differences are due to coupling between meta-atoms, fabrication errors (e.g. variation and scratches in the PMMA spacer), and residual interference from stray reflections from the experimental setup.

Major recent advances in high frequency electronics, and receiver and transmit system integration have now reached a price-point where it is commercially viable to exploit a large part of this spectrum for consumer applications, such as the increased information transfer envisaged for next generation mobile telephone communications ${ }^{46}$, or the new capability provided by single-chip radar for imaging or diagnostic systems, e.g. for security systems, gesture recognition, materials identification, car radar, robotic applications and autonomous vehicles $^{47,48}$. A key challenge for the realization of this roadmap is the development of low cost, low weight, reflect, or transmit antennas that can be scaled to large areas to provide directional, high quality beams. One of the eventual goals of this work is the development of large flexible holographic films at mm-wave frequencies that would offer the advantage of easy retrofitting to existing design structures whilst providing a specific tailored scattering or focussing response to an incoming beam. For example, coating walls, ceilings, windows or large flat panels with such antennas has scope to significantly improve the reliability of information transfer between high bandwidth mm-wave mobile devices and local base-stations in large rooms or halls, where beam blockage at high mm-wave frequencies is expected to be a major issue. There is also scope to provide planar high gain antennas for line of sight communication links, as well as smaller systems targeting specific radar applications.

In conclusion, we have created a platform for HMSs at a wavelength of $\lambda=3.19 \mathrm{~mm}$ with both rigid and flexible implementations. This flexible implementation could be exploited for smart HMS patches to enhance and extend existing millimeter wavelength equipment for applications in electromagnetic shielding and antennas. The research data supporting this publication can be accessed at https://doi.org/ 10.17630/162a371-10c7-4512-b678-558951c76e55.
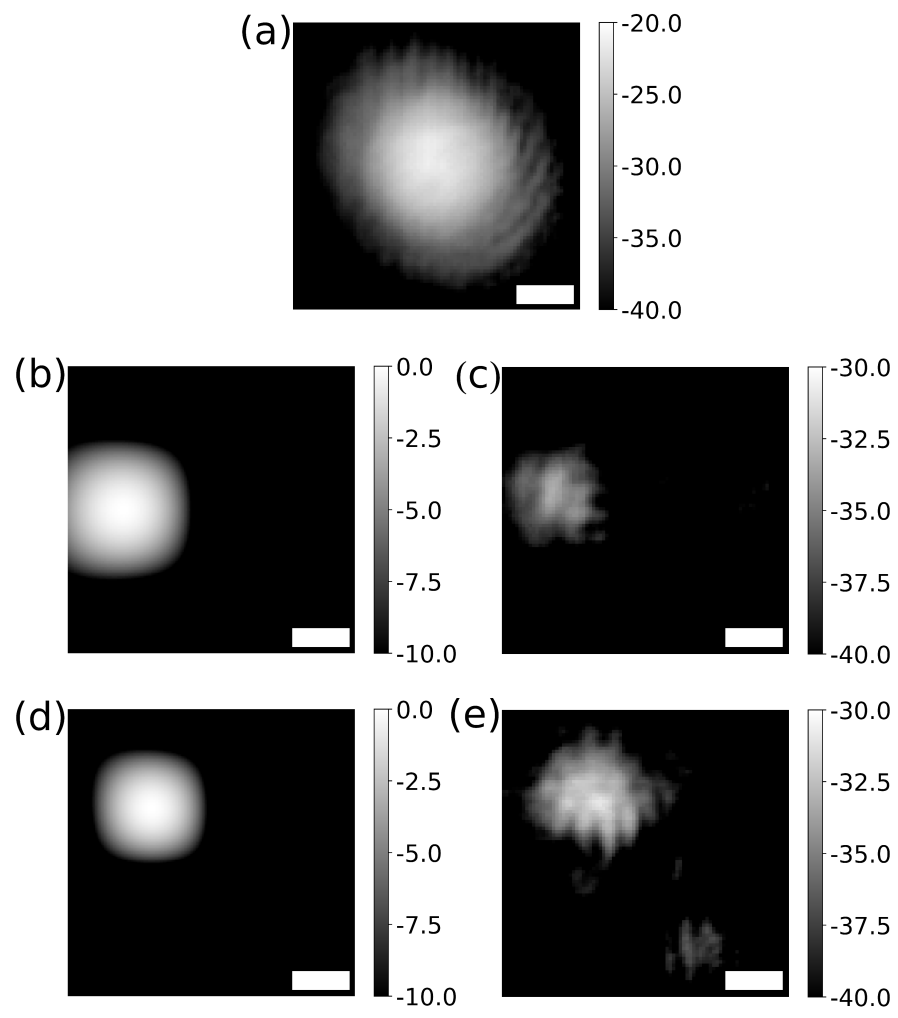

FIG. 5. (a) Displays the experimental image given by a polarization rotation mirror, in this case from linearly polarized along $x$ to linearly polarized along $y$. This represents the reference beam with rotated linear polarization. (b) Simulated and (c) experimental holographic images for the rigid HMS implementation deflected to one side. (d) Simulated and (e) experimental holographic images for the flexible HMS implementation deflected to a corner. All units for simulated images are on a normalized linear scale. All units for experimental images are in $\mathrm{dBm}$. The scale bar is the same for each image and represents $20 \mathrm{~mm}$.

This work was supported by DSTL (DASA grant ACC6004053). J.B. and J.M. contributed equally to this work. J.B. and A.D.F. acknowledge support from EPSRC (Grant Nos. EP/M508214/1 and EP/L017008/1). A.D.F is supported by the European Research Council (ERC) under the European Union Horizon 2020 research and innovation programme (grant agreement No. 819346).

\footnotetext{
${ }^{1}$ G. Zheng, H. Mühlenbernd, M. Kenney, G. Li, T. Zentgraf, and S. Zhang, "Metasurface holograms reaching 80\% efficiency," Nature Nanotechnology 10, 308-312 (2015)

${ }^{2}$ Z. Wei, Y. Cao, X. Su, Z. Gong, Y. Long, and H. Li, "Highly efficient beam steering with a transparent metasurface," Optics Express 21, 10739-10745 (2013).

${ }^{3}$ A. Arbabi, Y. Horie, A. Ball, M. Bagheri, and A. Faraon, "Subwavelengththick lenses with high numerical apertures and large efficiency based on high-contrast transmitarrays," Nature Communications 6, 7069 (2015).

${ }^{4}$ N. Yu and F. Capasso, "Flat optics with designer metasurfaces," Nature Materials 13, 139-150 (2014).

${ }^{5}$ M. Khorasaninejad, W. T. Chen, R. C. Devlin, J. Oh, A. Y. Zhu, and F. Capasso, "Metalenses at visible wavelengths: Diffraction-limited focusing and subwavelength resolution imaging," Science 352, 1190-1194 (2016).

${ }^{6}$ J. Cheng, S. Jafar-Zanjani, and H. Mosallaei, "All-dielectric ultrathin con-
} 
formal metasurfaces: lensing and cloaking applications at $532 \mathrm{~nm}$ wavelength," Scientific Reports 6, 38440 (2016).

${ }^{7}$ D. Wen, F. Yue, G. Li, G. Zheng, K. Chan, S. Chen, M. Chen, K. F. Li, P. W. H. Wong, K. W. Cheah, E. Y. B. Pun, S. Zhang, and X. Chen, "Helicity multiplexed broadband metasurface holograms," Nature Communications 6, 8241 (2015).

${ }^{8}$ Y. Montelongo, J. Tenorio-Pearl, W. Milne, and T. Wilkinson, "Polarization switchable diffraction based on subwavelength plasmonic nanoantennas," Nano Letters 14, 294-298 (2013).

${ }^{9}$ H.-S. Ee and R. Agarwal, "Tunable metasurface and flat optical zoom lens on a stretchable substrate," Nano Letters 16, 2818-2823 (2016).

${ }^{10}$ S. C. Malek, H.-S. Ee, and R. Agarwal, "Strain multiplexed metasurface holograms on a stretchable substrate," Nano Letters 17, 3641-3645 (2017).

${ }^{11}$ J. Burch, D. Wen, C. Xianzhong, and A. Di Falco, "Conformable holographic metasurfaces," Scientific Reports 7, 4520 (2017).

${ }^{12}$ L. Huang, H. Mühlenbernd, X. Li, X. Song, B. Bai, Y. Wang, and T. Zentgraf, "Broadband hybrid holographic multiplexing with geometric metasurfaces," Advanced Materials 27, 6444-6449 (2015).

${ }^{13}$ W. Ye, F. Zeuner, X. Li, B. Reineke, S. He, C.-W. Qiu, J. Liu, Y. Wang, S. Zhang, and T. Zentgraf, "Spin and wavelength multiplexed nonlinear metasurface holography," Nature Communications 7, 11930 (2016).

${ }^{14}$ S. M. Kamali, E. Arbabi, A. Arbabi, Y. Horie, M. Faraji-Dana, and A. Faraon, "Angle-multiplexed metasurfaces: encoding independent wavefronts in a single metasurface under different illumination angles," Phys Rev X 7, 041056 (2017).

${ }^{15}$ D. Wen, S. Chen, F. Yue, K. Chan, M. Chen, M. Ardron, K. F. Li, P. W. H. Wong, K. W. Cheah, E. Y. B. Pun, G. Li, S. Zhang, and X. Chen, "Metasurface device with helicity-dependent functionality," Advanced Optical Materials 4, 321-327 (2016).

${ }^{16}$ L. Huang, X. Chen, H. Mühlenbernd, H. Zhang, S. Chen, B. Bai, Q. Tan, G. Jin, K.-W. Cheah, C.-W. Qiu, J. Li, T. Zentgraf, and S. Zhang, "Threedimensional optical holography using a plasmonic metasurface," Nature Communications 4, 2808 (2013).

${ }^{17}$ S. Larouche, Y.-J. Tsai, T. Tyler, N. M. Jokerst, and D. R. Smith, "Infrared metamaterial phase holograms," Nature materials 11, 450 (2012).

${ }^{18}$ Y.-W. Huang, W. T. Chen, W.-Y. Tsai, P. C. Wu, C.-M. Wang, G. Sun, and D. P. Tsai, "Aluminum plasmonic multicolor meta-hologram," Nano Letters 15, 3122-3127 (2015).

${ }^{19}$ Y. Deng, X. Wang, Z. Gong, K. Dong, S. Lou, N. Pégard, K. B. Tom, F. Yang, Z. You, L. Waller, et al., "All-silicon broadband ultraviolet metasurfaces," Advanced Materials 30, 1802632 (2018).

${ }^{20}$ J. Wang, J. Ma, Z. Shu, Z.-D. Hu, and X. Wu, "Terahertz metalens for multifocusing bidirectional arrangement in different dimensions," IEEE Photonics Journal 11, 1-11 (2019).

${ }^{21}$ Q. Yang, J. Gu, D. Wang, X. Zhang, Z. Tian, C. Ouyang, R. Singh, J. Han, and W. Zhang, "Efficient flat metasurface lens for terahertz imaging," Opt. Express 22, 25931-25939 (2014).

${ }^{22}$ Q. Wang, X. Zhang, Y. Xu, Z. Tian, J. Gu, W. Yue, S. Zhang, J. Han, and W. Zhang, "A broadband metasurface-based terahertz flat-lens array," Advanced Optical Materials 3, 779-785 (2015).

${ }^{23}$ R. Singh, W. Cao, I. Al-Naib, L. Cong, W. Withayachumnankul, and W. Zhang, "Ultrasensitive terahertz sensing with high-q fano resonances in metasurfaces," Applied Physics Letters 105, 171101 (2014).

${ }^{24}$ L. Cong, S. Tan, R. Yahiaoui, F. Yan, W. Zhang, and R. Singh, "Experimental demonstration of ultrasensitive sensing with terahertz metamaterial absorbers: A comparison with the metasurfaces," Applied Physics Letters 106, 031107 (2015).

${ }^{25}$ Y. Fan, N.-H. Shen, T. Koschny, and C. M. Soukoulis, "Tunable terahertz meta-surface with graphene cut-wires," Acs Photonics 2, 151-156 (2015).

${ }^{26}$ D. Wang, L. Zhang, Y. Gu, M. Mehmood, Y. Gong, A. Srivastava, L. Jian, T. Venkatesan, C.-W. Qiu, and M. Hong, "Switchable ultrathin quarterwave plate in terahertz using active phase-change metasurface," Scientific reports 5, 15020 (2015).
${ }^{27}$ H. Zhu, S. Cheung, X. Liu, and T. Yuk, "Design of polarization reconfigurable antenna using metasurface," IEEE transactions on antennas and propagation 62, 2891-2898 (2014).

${ }^{28}$ H. Zhu, X. Liu, S. Cheung, and T. Yuk, "Frequency-reconfigurable antenna using metasurface," IEEE Transactions on Antennas and Propagation 62 , 80-85 (2014).

${ }^{29}$ H. L. Zhu, S. W. Cheung, and T. I. Yuk, "Mechanically pattern reconfigurable antenna using metasurface," IET Microwaves, Antennas \& Propagation 9, 1331-1336 (2015).

${ }^{30}$ P.-Y. Chen, J. Soric, Y. R. Padooru, H. M. Bernety, A. B. Yakovlev, and A. Alù, "Nanostructured graphene metasurface for tunable terahertz cloaking," New Journal of Physics 15, 123029 (2013).

${ }^{31}$ Y.-C. Song, J. Ding, C.-J. Guo, Y.-H. Ren, and J.-K. Zhang, "Ultrabroadband backscatter radar cross section reduction based on polarizationinsensitive metasurface," IEEE Antennas and Wireless Propagation Letters 15, 329-331 (2016).

${ }^{32}$ B. Orazbayev, N. Mohammadi Estakhri, A. Alù, and M. Beruete, "Experimental demonstration of metasurface-based ultrathin carpet cloaks for millimeter waves," Advanced Optical Materials 5, 1600606 (2017).

${ }^{33}$ L. Liu, X. Zhang, M. Kenney, X. Su, N. Xu, C. Ouyang, Y. Shi, J. Han, W. Zhang, and S. Zhang, "Broadband metasurfaces with simultaneous control of phase and amplitude," Advanced Materials 26, 5031-5036 (2014).

${ }^{34} \mathrm{C}$. Pfeiffer and A. Grbic, "Millimeter-wave transmitarrays for wavefront and polarization control," IEEE Transactions on Microwave Theory and Techniques 61, 4407-4417 (2013).

${ }^{35}$ X. Liu, K. Fan, I. V. Shadrivov, and W. J. Padilla, "Experimental realization of a terahertz all-dielectric metasurface absorber," Optics express 25, 191201 (2017).

${ }^{36}$ H. Zhu, S. Cheung, K. L. Chung, and T. I. Yuk, "Linear-to-circular polarization conversion using metasurface," IEEE Transactions on Antennas and Propagation 61, 4615-4623 (2013).

${ }^{37}$ N. W. Caira and D. R. Smith, "Multispectral metasurface hologram at millimeter wavelengths," Applied optics 57, A19-A25 (2018).

${ }^{38}$ Z. H. Jiang, L. Kang, W. Hong, and D. H. Werner, "Highly efficient broadband multiplexed millimeter-wave vortices from metasurface-enabled transmit-arrays of subwavelength thickness," Physical Review Applied 9, 064009 (2018).

${ }^{39} \mathrm{R}$. Gerchberg, "A practical algorithm for the determination of phase from image and diffraction plane pictures," Optik 35, 237 (1972).

${ }^{40} \mathrm{~J}$. Goodman, Introduction to Fourier optics (McGraw-hill, 2008).

${ }^{41}$ J. A. Veerman, J. J. Rusch, and H. P. Urbach, "Calculation of the rayleighsommerfeld diffraction integral by exact integration of the fast oscillating factor," JOSA A 22, 636-646 (2005).

${ }^{42}$ J. Burch and A. Di Falco, "Surface topology specific metasurface holograms," ACS Photonics 5, 1762-1766 (2018).

${ }^{43}$ J. Burch and A. Di Falco, "Holography using curved metasurfaces," in Photonics, Vol. 6 (Multidisciplinary Digital Publishing Institute, 2019) pp. 1-8.

${ }^{44}$ N. Kooy, K. Mohamed, L. T. Pin, and O. S. Guan, "A review of roll-to-roll nanoimprint lithography," Nanoscale research letters 9, 320 (2014).

${ }^{45}$ S. M. Kamali, A. Arbabi, E. Arbabi, Y. Horie, and A. Faraon, "Decoupling optical function and geometrical form using conformal flexible dielectric metasurfaces," Nature communications 7, 11618 (2016).

${ }^{46}$ T. S. Rappaport, S. Sun, R. Mayzus, H. Zhao, Y. Azar, K. Wang, G. N. Wong, J. K. Schulz, M. Samimi, and F. Gutierrez, "Millimeter wave mobile communications for 5g cellular: It will work!" IEEE access 1, 335-349 (2013).

${ }^{47}$ J. du Preez and S. Sinha, "Practical applications of millimeter-wave antennas," in Millimeter-Wave Antennas: Configurations and Applications (Springer, 2016) pp. 133-155.

${ }^{48}$ S. Dhillon, M. Vitiello, E. Linfield, A. Davies, M. C. Hoffmann, J. Booske, C. Paoloni, M. Gensch, P. Weightman, G. Williams, et al., "The 2017 terahertz science and technology roadmap," Journal of Physics D: Applied Physics 50, 043001 (2017). 